\title{
Minimally invasive anterior and lateral transpsoas approaches for closed reduction of grade II spondylolisthesis: initial clinical and radiographic experience
}

\author{
David S. Xu, MD, ${ }^{1}$ Konrad Bach, MD, ${ }^{2}$ and Juan S. Uribe, MD${ }^{1}$ \\ 1'Division of Spinal Disorders, Department of Neurological Surgery, Barrow Neurological Institute, St. Joseph's Hospital and \\ Medical Center, Phoenix, Arizona; and '2Division of Spine, Department of Neurosurgery and Brain Repair, University of South \\ Florida, Tampa, Florida
}

OBJECTIVE Minimally invasive anterior and lateral approaches to the lumbar spine are increasingly used to treat and reduce grade I spondylolisthesis, but concerns still exist for their usage in the management of higher-grade lesions. The authors report their experience with this strategy for grade II spondylolisthesis in a single-surgeon case series and provide early clinical and radiographic outcomes.

METHODS A retrospective review of a single surgeon's cases between 2012 and 2016 identified all patients with a Meyerding grade II lumbar spondylolisthesis who underwent minimally invasive lateral lumbar interbody fusion (LLIF) or anterior lumbar interbody fusion (ALIF) targeting the slipped level. Demographic, clinical, and radiographic data were collected and analyzed. Changes in radiographic measurements, Oswestry Disability Index (ODI), and visual analog scale (VAS) scores were compared using the paired t-test and Wilcoxon signed rank test for continuous and ordinal variables, respectively.

RESULTS The average operative time was 199.1 minutes (with $60.6 \mathrm{ml}$ of estimated blood loss) for LLIFs and 282.1 minutes (with $106.3 \mathrm{ml}$ of estimated blood loss), for ALIFs. Three LLIF patients had transient unilateral anterior thigh numbness during the 1st week after surgery, and $1 \mathrm{ALIF}$ patient had transient dorsiflexion weakness, which was resolved at postoperative week 1. The mean follow-up time was 17.6 months (SD 12.5 months) for LLIF patients and 10 months (SD 3.1 months) for ALIF patients. Complete reduction of the spondylolisthesis was achieved in 12 LLIF patients $(75.0 \%)$ and 7 ALIF patients (87.5\%). Across both procedures, there was an increase in both the segmental lordosis (LLIF 5.6, $\mathrm{p}$ $=0.002$; ALIF $15.0^{\circ}, p=0.002$ ) and overall lumbar lordosis (LLIF 2.9,$p=0.151 ;$ ALIF $5.1^{\circ}, p=0.006$ ) after surgery. Statistically significant decreases in the mean VAS and the mean ODI measurements were seen in both treatment groups. The VAS and ODI scores fell by a mean value of $3.9(p=0.002)$ and $19.8(p=0.001)$, respectively, for LLIF patients and $3.8(p=0.02)$ and $21.0(p=0.03)$, respectively, for ALIF patients at last follow-up.

CONCLUSIONS Early clinical and radiographic results from using minimally invasive LLIF and ALIF approaches to treat grade II spondylolisthesis appear to be good, with low operative blood loss and no neurological deficits. Complete reduction of the spondylolisthesis is frequently possible with a statistically significant reduction in pain scores.

https://thejns.org/doi/abs/10.3171/2017.10.FOCUS17574

KEY WORDS MIS; ALIF; LIF; XLIF; spondylolisthesis

$\mathrm{P}$ ATIENTS with higher-grade lumbar spondylolisthesis (Meyerding grade II and above) are typically chosen as surgical candidates due to severe axial back pain with associated radiculopathy, neurogenic claudication, segmental deformity, or focal instability. The goals of operative intervention therefore center on decompressing neural structures, providing adequate fixation, and restoring spinopelvic alignment. ${ }^{5,14,15,20}$ Traditional open or minimally invasive (MIS) transforaminal lumbar interbody fusion (TLIF) and posterior lumbar interbody fusion

ABBREVIATIONS ALIF = anterior lumbar interbody fusion; LLIF = lateral lumbar interbody fusion; MIS = minimally invasive; ODI = Oswestry Disability Index; PEEK = polyetheretherketone; PLIF = posterior lumbar interbody fusion; TLIF = transforaminal lumbar interbody fusion; VAS = visual analog scale (for pain).

SUBMITTED September 1, 2017. ACCEPTED October 17, 2017.

INCLUDE WHEN CITING DOI: 10.3171/2017.10.FOCUS17574. 
(PLIF) approaches have been routinely used to treat higher-grade spondylolisthesis with good results..$^{8,23,25}$ In the setting of degenerative spondylolisthesis, MIS lateral and anterior approaches to the lumbar spine have also demonstrated clinical and radiographic outcomes equivalent to those of posterior approaches, ${ }^{18,30}$ but they offer potential biomechanical advantages, such as placement of a larger interbody, thus allocating more surface area for arthrodesis while also enabling greater restoration of lumbar lordosis. ${ }^{22}$ Furthermore, the indirect foraminal and central canal decompression generated by placement of a large interbody may negate the need for a posterior decompression, avoiding disruption of osseous and ligamentous elements. $^{12}$

Despite these advantages, many surgeons harbor reservations about utilizing MIS lateral and anterior approaches for treating higher-grade spondylolisthesis. Concerns center around risk to the lumbar plexus when traversing through the psoas muscle, traction injury to nerve roots when reducing vertebral alignment without first exposing the course of the nerve root, and inadequate decompression of the neuroforamen or thecal sac when relying on only indirect decompression through restoration of the disc height. For lateral approaches, there is additional concern when addressing the L4-5 level due to the lumbar plexus residing in its most ventral anatomical location, raising the risk of retraction injury to the femoral nerve, which may be further exacerbated in the setting of spondylolisthesis. ${ }^{1,3}$ Unfortunately, the experience reported in the literature is limited with regard to these concerns, and few studies provide detailed radiographic outcomes with regard to spinopelvic parameters.

In this study, we present the initial clinical and radiographic outcomes of using an MIS anterior lumbar interbody fusion (ALIF) and lateral transpsoas lumbar interbody fusion (LLIF) in conjunction with posterior percutaneous pedicle screws without direct decompression for the treatment of grade II spondylolisthesis. Our data reveal that thorough knowledge of regional retroperitoneal anatomy and thoughtful application of contemporary intraoperative electromyographic neuromonitoring enable safe treatment of higher-grade spondylolisthesis through an MIS anterior or lateral corridor with low morbidity and good initial patient outcomes.

\section{Methods \\ Patient Population}

A retrospective review of a single surgeon's prospectively maintained operative database identified all consecutive patients with a lumbar spondylolisthesis of Meyerding grade II who underwent an MIS LLIF or ALIF targeting the affected level between 2012 and 2016. All patients had intractable back pain coexisting with either neurogenic claudication or radiculopathy that did not respond to conservative measures. No patients had previous lumbar surgeries, history of significant trauma, or significant coronal or sagittal deformity. Treatment strategies consisted of segmental interbody fusion at the level of spondylolisthesis combined with percutaneous short-segment pedicle screw fixation without posterior decompression.

\section{Data Collection}

The clinical data consisted of visual analog scale (VAS) and Oswestry Disability Index (ODI) scores, estimated periprocedural blood loss, length of surgery, and length of stay. Radiographic measurements of the degrees of anterolisthesis, segmental lordosis, and overall lumbar lordosis were obtained from standing radiographs preoperatively and at the most recent follow-up. Fusion status was assessed for all patients with at least 1 year of imaging follow-up; plain radiographs obtained at the 1-year follow-up visit were reviewed by all authors to verify fusion, which was defined as the presence of bridging bone across the disc space (Bridwell grade 1 or 2).,19 All data were collected in conjunction with an IRB-approved research protocol with waiver of patient consent.

\section{Surgical Procedure}

For patients treated with a lateral approach, we used the extreme lateral interbody fusion procedure (XLIF, NuVasive). Briefly, an MIS lateral retroperitoneal transpsoas approach was used to expose the lumbar spine. Free-running directional electromyography was then used to define a corridor through the psoas muscle anterior to the lumbar plexus and femoral nerve. A working retractor was docked in the disc space as posterior as possible to the superior vertebral body while ensuring that the neuroforamen of the interspace was not violated (Fig. 1). An aggressive discectomy was performed, and a $10^{\circ}$ lordotic $18-26 \times$ $8-10 \times 50-60-\mathrm{mm}$ polyetheretherketone (PEEK) interbody graft was placed.

All anterior approaches used a mini-open transrectus retroperitoneal approach performed by a vascular approach surgeon. Briefly, a small paramedian incision was made at the level of the anterior superior iliac spine. The lateral aponeurosis of the rectus muscle was incised, and the posterior transversalis fascia was divided, allowing entry into the retroperitoneal space. After the great vessels ventral to the lumbar spine were mobilized laterally, the targeted interspace was treated with a discectomy followed by sizing and placement of a large PEEK cage.

In most patients, placement of the interbody alone resulted in approximately 50\% reduction of the spondylolisthesis. After interbody placement, all patients were positioned prone and percutaneous pedicle screws were placed above and below the treated level. Rod fixation then proceeded first by insertion and final tightening in the inferior pedicle screws, followed by cantilever reduction of the superior pedicle screws into the rod, which allowed further reduction of the spondylolisthesis.

\section{Statistical Analysis}

The paired t-test and Wilcoxon signed-rank test were used to analyze changes in continuous and ordinal clinical variables, respectively; $\mathrm{p}<0.05$ was considered to indicate statistical significance.

\section{Results}

\section{Patient Population}

A total of 28 patients with grade II spondylolisthesis 

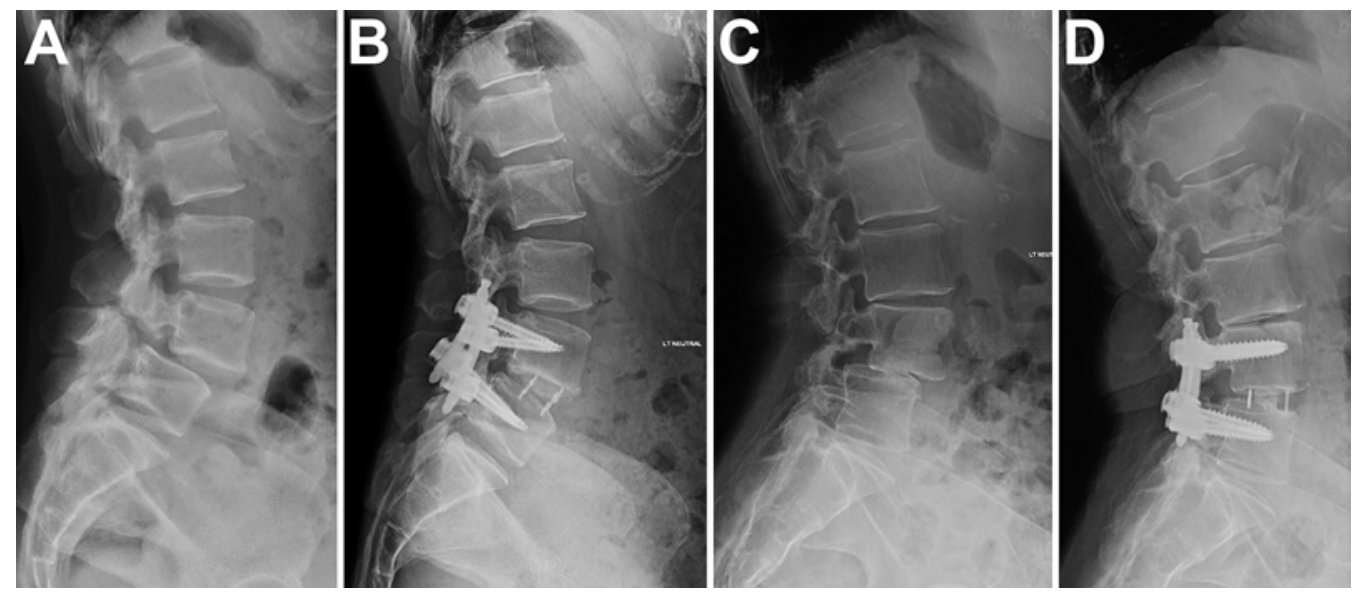

FIG. 1. A and B: Case 15. Lateral radiographs obtained before (A) and 3 months after (B) LLIF reduction of a grade II L4-5 spondylolisthesis. C and D: Case 13. Lateral radiographs obtained before (C) and after (D) the same procedure at the same level. In both cases, complete reduction of the spondylolisthesis was achieved without complication.

were identified as having been treated during the 4-year study period. Twenty-four of these patients underwent either an MIS LLIF or ALIF procedure and were included in this study; their demographic and operative data are outlined in Table 1 . This study group included 8 men and 16 women; the patients' mean age was 55.6 years. All 24 patients harbored a grade II spondylolisthesis measured on preoperative CT images, with the level of slippage occurring at L3-4 in 1 patient, at L4-5 in 15 patients, and at L5-S1 in 8 patients. All patients with spondylolisthesis above L-5 underwent LLIF (Fig. 1), and all patients with slippage at L5-S1 underwent ALIF (Fig. 2). Four other patients underwent a posterior MIS TLIF. Information was unavailable regarding any additional patients seen who did not undergo surgery due to low bone-mineral density or choice of pursuing conservative management.

\section{Operative Data and Complications}

In the LLIF group, the average operative time was 199.1 minutes, with $60.6 \mathrm{ml}$ of estimated blood loss and an average length of stay of 2.2 days (median 2 days). In the ALIF group, the average operative time was 282.1 minutes, with $106.3 \mathrm{ml}$ of estimated blood loss and an average length of stay of 4.3 days (median 4.5) (Table 1). Three patients in the LLIF group had transient unilateral anterior thigh numbness that resolved within a week after surgery, and 1 patient treated with ALIF had transient dorsiflexion weakness that also resolved within the first postoperative week. No patient had any persistent motor or sensory deficit after surgery.

\section{Radiographic Outcomes}

The mean follow-up time was 17.6 months (SD 12.6 months) for LLIF patients and 10 months (SD 3.1 months) for ALIF patients, with $62.5 \%$ of both patient groups having follow-up of at least 1 year. The outcome measures are summarized in Table 2. Complete reduction of the spondylolisthesis was achieved in $12(75.0 \%)$ of the patients treated with LLIF and 7 (87.5\%) of those treated with ALIF (Table 1). Across both groups, there was an increase in both the segmental lordosis (LLIF $5.6^{\circ}, \mathrm{p}=0.002$; ALIF $15.0^{\circ}, \mathrm{p}=0.002$ ) and overall lumbar lordosis (LLIF $2.9^{\circ}, \mathrm{p}$ $=0.151$; ALIF 5.1 $\left.1^{\circ}, \mathrm{p}=0.006\right)$ after surgery. All patients $(\mathrm{n}$ $=15$ ) with imaging follow-up past 1 year showed evidence of bony fusion across the implant at 1 year postsurgery.

\section{Clinical Outcomes}

A statistically significant decrease in both the mean VAS and mean ODI scores was seen in both treatment groups (Table 2). For LLIF patients, the mean decreases in VAS and ODI scores were $3.9(\mathrm{p}=0.002)$ and $19.8(\mathrm{p}=$ $0.001)$, respectively, and for ALIF they were $3.8(\mathrm{p}=0.02)$ and $21.0(\mathrm{p}=0.03)$, respectively, at the time of last followup. No patient required repeat operative intervention or revision of hardware during follow-up.

\section{Discussion}

Open and MIS posterior decompression and reduction of spondylolisthesis are well described in the literature and have demonstrated satisfactory patient outcomes, with MIS approaches associated with shorter length of stay, reduced hospital charges, and lower frequency of patient transfers to acute rehabilitation postoperatively. ${ }^{16,25,31}$ Reports of LLIF and ALIF approaches for the treatment of grade I spondylolisthesis are less common but demonstrate outcomes comparable with those achieved with posterior MIS approaches. Marchi et al..$^{21}$ reported outcomes from using a stand-alone LLIF cage in 52 patients and found slippage reduction rates exceeding $90 \%$, with significant reduction of VAS and ODI scores at 1 year and no instances of persistent neurological deficits. Ahmadian et al. ${ }^{1}$ reported on a cohort of 26 patients with grade I and 5 patients with grade II L4-5 spondylolisthesis treated with LLIF and posterior percutaneous pedicle screws and found that full reduction of slippage could be achieved in $87 \%$ of patients, with significant reduction in VAS scores and no permanent neurological deficit. Lee et al. ${ }^{18}$ performed a mini-open ALIF with percutaneous pedicle screws in 46 patients with grade I L5-S1 spondylolisthesis and 27 patients with grade II slippage with no incidence of neu- 
TABLE 1. Patient demographic and operative data

\begin{tabular}{|c|c|c|c|c|c|c|c|c|c|c|}
\hline $\begin{array}{l}\text { Procedure } \\
\& \text { Case No. }\end{array}$ & $\begin{array}{l}\text { Age } \\
\text { (yrs) }\end{array}$ & Sex & $\begin{array}{l}\text { Level of } \\
\text { Slippage }\end{array}$ & $\begin{array}{l}\text { Meyerding } \\
\text { Grade }\end{array}$ & $\begin{array}{l}\text { Complete } \\
\text { Reduction }\end{array}$ & $\begin{array}{c}\mathrm{FU} \\
\text { (mos) }\end{array}$ & $\begin{array}{l}\text { Op Time } \\
\text { (mins) }\end{array}$ & $\mathrm{EBL}(\mathrm{ml})$ & $\begin{array}{l}\text { Length of } \\
\text { Stay (days) }\end{array}$ & Adverse Event \\
\hline \multicolumn{11}{|l|}{ LLIF } \\
\hline 1 & 73 & $\mathrm{~F}$ & L3-4 & 2 & Yes & 43 & 192 & 70 & 2 & \\
\hline 2 & 40 & $\mathrm{~F}$ & L4-5 & 2 & No & 15 & 249 & 50 & 1 & Transient ant thigh numbness \\
\hline 3 & 65 & $\mathrm{~F}$ & L4-5 & 2 & Yes & 29 & 231 & 25 & 2 & \\
\hline 4 & 73 & $\mathrm{~F}$ & L4-5 & 2 & Yes & 25 & 224 & 100 & 1 & \\
\hline 5 & 70 & $\mathrm{~F}$ & L4-5 & 2 & No & 27 & 176 & 25 & 2 & \\
\hline 6 & 63 & M & L4-5 & 2 & Yes & 29 & 258 & 200 & 5 & Transient ant thigh numbness \\
\hline 7 & 39 & $\mathrm{~F}$ & L4-5 & 2 & Yes & 20 & 189 & 15 & 5 & \\
\hline 8 & 58 & $\mathrm{~F}$ & L4-5 & 2 & No & 6 & 181 & 50 & 2 & \\
\hline 9 & 51 & M & L4-5 & 2 & Yes & 3 & 207 & 50 & 1 & \\
\hline 10 & 60 & M & L4-5 & 2 & Yes & 29 & 230 & 50 & 1 & \\
\hline 11 & 71 & $\mathrm{~F}$ & L4-5 & 2 & No & 3 & 161 & 50 & 3 & Transient ant thigh numbness \\
\hline 12 & 53 & $\mathrm{~F}$ & L4-5 & 2 & Yes & 15 & 194 & 50 & 2 & \\
\hline 13 & 58 & M & L4-5 & 2 & Yes & 26 & 185 & 50 & 1 & \\
\hline 14 & 45 & $M$ & L4-5 & 2 & Yes & 6 & 204 & 15 & 3 & \\
\hline 15 & 47 & $\mathrm{~F}$ & L4-5 & 2 & Yes & 3 & 156 & 20 & 1 & \\
\hline 16 & 73 & $\mathrm{~F}$ & L4-5 & 2 & Yes & 3 & 148 & 150 & 3 & \\
\hline Mean (SD) & & & & & & $17.6(12.6)$ & $199.1(32.5)$ & $60.6(50.5)$ & $2.2(1.3)$ & \\
\hline \multicolumn{11}{|l|}{ ALIF } \\
\hline 17 & 54 & $\mathrm{~F}$ & L5-S1 & 2 & No & 12 & 360 & 30 & 4 & \\
\hline 18 & 37 & $\mathrm{~F}$ & L5-S1 & 2 & Yes & 12 & 305 & 50 & 5 & \\
\hline 19 & 66 & $\mathrm{~F}$ & L5-S1 & 2 & Yes & 12 & 301 & 150 & 3 & \\
\hline 20 & 49 & $\mathrm{~F}$ & L5-S1 & 2 & Yes & 12 & 319 & 200 & 2 & \\
\hline 21 & 54 & M & L5-S1 & 2 & Yes & 7 & 328 & 100 & 5 & \\
\hline 22 & 53 & M & L5-S1 & 2 & Yes & 6 & 250 & 20 & 6 & \\
\hline 23 & 28 & $\mathrm{~F}$ & L5-S1 & 2 & Yes & 6 & 242 & 200 & 4 & \\
\hline 24 & 55 & $M$ & L5-S1 & 2 & Yes & 13 & 152 & 100 & 5 & Transient dorsiflexion weakness \\
\hline Mean (SD) & & & & & & $10(3.1)$ & $282.1(65.4)$ & $106.3(71.7)$ & $4.3(1.3)$ & \\
\hline
\end{tabular}

Ant $=$ anterior $; \mathrm{EBL}=$ estimated blood loss $; \mathrm{FU}=$ follow-up.

rological deficit, but only limited pain and radiographic outcomes were presented.

Few reports are available regarding application of MIS techniques for closed reduction of grade II or higher spondylolisthesis. To our knowledge, a recent article by Rajakumar et al. ${ }^{27}$ remains the only large case series in the literature examining the use of MIS TLIF for the treatment of grade II and III slippages, with excellent reported outcomes. Our perioperative results are similar with respect to average blood loss (LLIF $60 \mathrm{ml}$ and ALIF 106 $\mathrm{ml}$ vs TLIF $90 \mathrm{ml}$ ) and length of stay (LLIF 2.2 days and ALIF 4.3 days vs TLIF 2-3 days), but we encountered longer operative times due to repositioning of the patient for pedicle screw placement (LLIF 199.1 minutes and ALIF 282.1 minutes vs TLIF 146.7 minutes). We believe that for higher-grade spondylolisthesis, as in the treatment of grade I spondylolisthesis, ${ }^{30}$ lateral and anterior MIS approaches are complimentary to posterior strategies by offering comparable reduced muscle disruption, avoidance of neural tissue manipulation, and lower blood loss and length of stay. Furthermore, in patients who have previously undergone posterior surgery, an anterior or lateral approach bypasses the morbidity imposed by navigating through scar tissue.

Within the literature, the bulk of grade II and highergrade lesions are included as small subsets of larger lateral and anterior MIS surgical cases series, ${ }^{2,18}$ with no study other than ours and the aforementioned paper by Rajakumar et al. ${ }^{27}$ evaluating a cohort limited to patients with higher-grade spondylolistheses. The limited amount of available data may be due not only to the rarity of higher-grade lesions, but also to the reluctance of surgeons to employ an operative strategy that relies on indirect decompression. Specifically, when considering anterior and lateral approaches for spondylolisthesis, 3 predominant concerns arise (described below).

\section{Exiting Nerve Root Injury}

Classically, risk of injury to the exiting nerve root at the level of the spondylolisthesis, especially at L-5, has been a major concern when considering reduction without full posterior decompression of the nerve root. ${ }^{10,13}$ The exact mechanism of injury is still poorly understood, but a study 

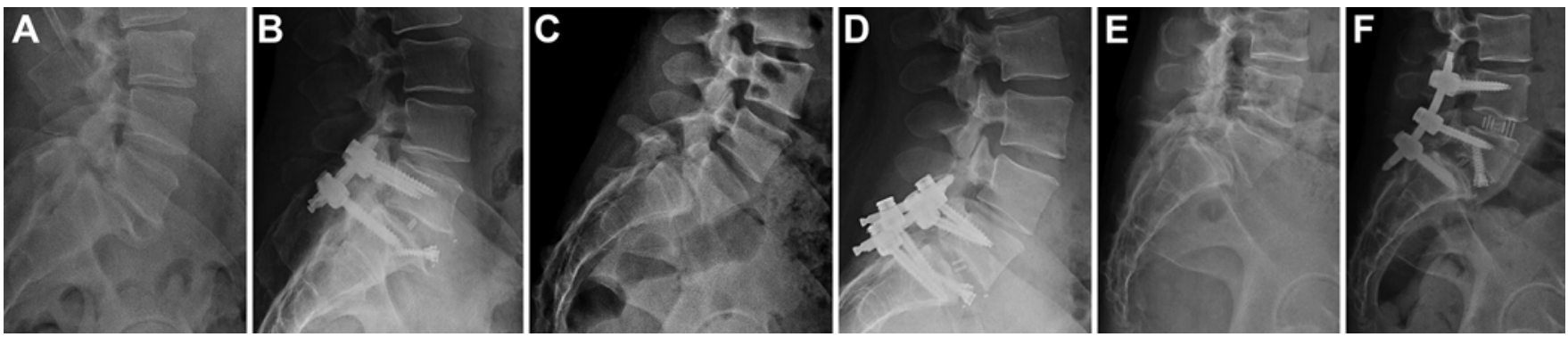

FIG. 2. A and B: Case 21. Lateral radiographs obtained before (A) and 3 months after (B) ALIF reduction of a grade II L5-S1 spondylolisthesis. The postoperative radiograph shows complete reduction of the slippage. C and D: Case 23. Similar lateral radiographs obtained in a patient with a grade II L5-S1 spondylolisthesis before (C) and after (D) the same procedure. The postoperative radiograph again demonstrates complete reduction. E and F: Case 17. Lateral preoperative $(E)$ and postoperative $(F)$ radiographs obtained in a patient who had a grade II L5-S1 spondylolisthesis. In the setting of a pelvic-incidence/lumbar lordosis mismatch only partial reduction at L5-S1 was achieved with an ALIF and therefore an LLIF was performed at L4-5 to deliver additional lordosis. All 3 patients recovered without complication.

by Petraco et al. ${ }^{26}$ examining tension along the L-5 nerve root during reduction of high-grade spondylolisthesis found that the nerve at baseline is stretched to higher tensions due to the anterior subluxation of the superior vertebra, which predisposes the nerve to stretch neuropraxia during interbody restoration of the anterior column, due to either distraction from the increased disc space height or increased lateral axial traction along the course of the nerve root as it is reduced posteriorly. Interestingly, their study found that the greatest amount of tension on the exiting nerve root occurs during the latter half of the reduction distance and that the degree of stretch during reduction is reduced with greater segmental lordosis.

These findings provide an anatomical basis to explain why we encountered no incidence of permanent nerve root injury in our study. Although placement of a large ALIF or LLIF interbody provides significant distraction of the disc space, we observed intraoperatively that the degree of reduction achieved by the interbody alone prior to posterior fixation does not exceed half of the existing slippage. Furthermore, in our cohort, we demonstrated that placement of the interbody significantly increases the segmental lordosis (LLIF 5.6 $6^{\circ}$ ALIF $15.0^{\circ}$ ), thereby decreasing the interval tension on the nerve root during reduction. Lastly, when the interbody is placed, ALIF and LLIF approaches do not rely on concurrent application of additional disc space or pedicle screw distraction to maximize the size of the implant placed.

\section{Lumbar Plexus Risk From Lateral Access at L4-5}

Significant anatomical and radiographic literature has documented the ventral migration of the lumbar plexus as it descends caudally, with the plexus being the most anterior along its course through the psoas muscle at L4-5.,11 This places the plexus most at risk for anterior retraction injury at L4-5, a risk that is heightened when a highergrade spondylolisthesis further pushes the retractor docking zone anteriorly. As a rule, when placing the retractor at the disc space, the bottom vertebra should be used for target referencing, with a goal of placing the center of the retractor at $50 \%$ of the lateral endplate length without breaching the neuroforamen with the posterior retractor blade (Fig. 3). However, our results show that with contin- uous directional EMG stimulation verifying placement of the retractor anterior to the femoral nerve, it is possible to safely approach and treat grade II spondylolisthesis with an LLIF even at L4-5.

Despite these findings, multiple studies continue to cite early LLIF experiences without the use of directional neuromonitoring as evidence that transpsoas approaches are high risk; others take the earlier studies completely out of context. ${ }^{4,9}$ One recent literature review has frequently been misreferenced to claim that motor weakness resulting from lumbar plexus injuries occurs in up to $33.6 \%$ of reported LLIF cases. ${ }^{1}$ However, the purpose of the review was to advocate for a standardized classification of neurological findings after LLIF, and the motor weakness figure encompassed patients with transient iliopsoas weakness that occurs as results of muscle dissection by the retractor. When examining large contemporary LLIF series of more than 500 cases, including treatment at L4-5, the incidence of motor complications as a result of lumbar plexus injury is well under $1 \%$. $^{2,29}$

\section{Efficacy of Indirect Decompression}

In the setting of spondylolisthesis, symptomatic patients

TABLE 2. Clinical and radiographic outcomes

\begin{tabular}{lcccc}
\hline Variable & Preop & Postop & $\Delta$ & $\mathrm{p}$ Value \\
\hline LLIF & & & & \\
\hline $\begin{array}{l}\text { Segmental } \\
\text { lordosis }\end{array}$ & $16.3^{\circ}\left(5.1^{\circ}\right)$ & $21.9^{\circ}\left(5.4^{\circ}\right)$ & $5.6^{\circ}\left(5.9^{\circ}\right)$ & 0.002 \\
\hline Lumbar lordosis & $55.7^{\circ}\left(9.2^{\circ}\right)$ & $58.6^{\circ}\left(8.8^{\circ}\right)$ & $2.9^{\circ}\left(7.3^{\circ}\right)$ & 0.151 \\
\hline VAS & $7.8^{(1.7)}$ & $3.8(2.8)$ & $-3.9(3.4)$ & 0.002 \\
\hline ODI & $54.2(13.3)$ & $34.4(21.6)$ & $-19.8(15.6)$ & 0.001 \\
\hline ALIF & & & & \\
\hline $\begin{array}{l}\text { Segmental } \\
\text { lordosis }\end{array}$ & $18.2^{\circ}\left(7.2^{\circ}\right)$ & $33.2^{\circ}\left(5.1^{\circ}\right)$ & $15.0^{\circ}\left(8.8^{\circ}\right)$ & 0.002 \\
\hline Lumbar lordosis & $60.5^{\circ}\left(16.4^{\circ}\right)$ & $65.6^{\circ}\left(16.2^{\circ}\right)$ & $5.1^{\circ}\left(3.7^{\circ}\right)$ & 0.006 \\
\hline VAS & $7.0(1.7)$ & $3.3(2.8)$ & $-3.8(2.8)$ & 0.020 \\
\hline ODI & $47.3^{(11.9)}$ & $26.3(15.4)$ & $-21.0(18.0)$ & 0.030 \\
\hline DDa & & &
\end{tabular}

Data are presented as mean (SD). 


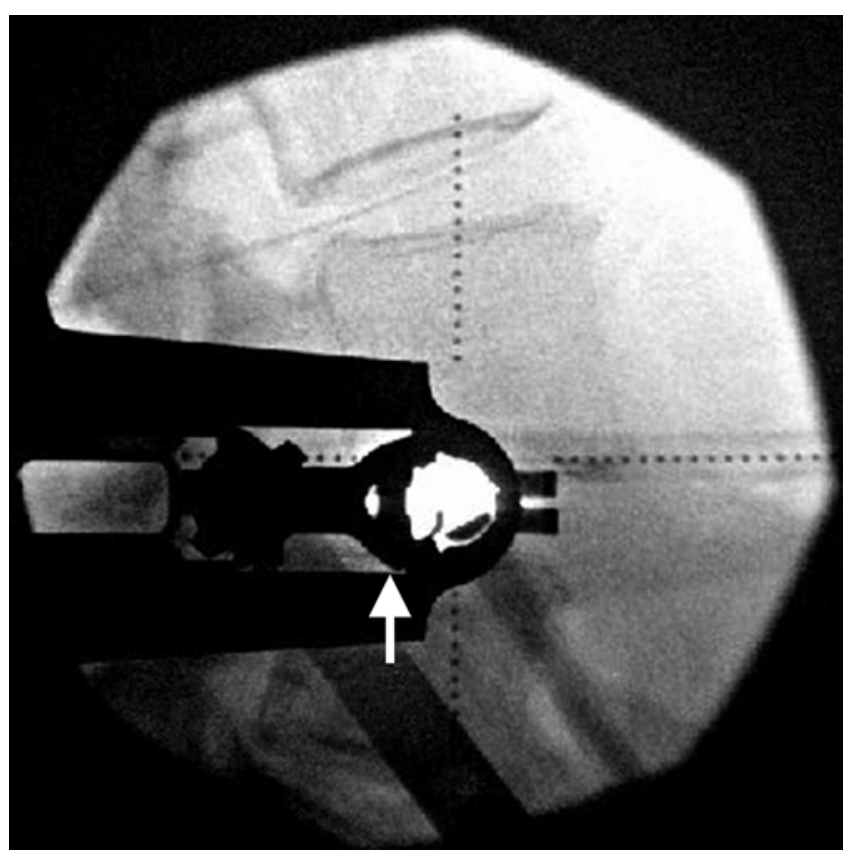

FIG. 3. Case 16. Lateral radiograph demonstrating ideal placement of the LLIF retractor. The inferior vertebra should be used for targeting reference with the goal to place the center of the retractor corridor at $50 \%$ of the length of the endplate. Care should be taken to ensure that the posterior blade of the retractor (arrow) does not encroach into the neuroforamen and that the nerve root course does not run through the field of the retractor or near the posterior blade where a docking shim is inserted into the disc space.

typically have significant central canal and neuroforaminal stenosis due to subluxation of the vertebral body as well as superimposed spondylitic changes and disc space collapse. In patients with higher-grade spondylolisthesis, the degree of stenosis may be even worse as increased vertebral body subluxation moves into the canal, prompting uncertainty as to whether indirect decompression will be adequate. Oliveira et al. ${ }^{24}$ performed volumetric analysis on pre- and postoperative lumbar spine MR images obtained in 21 patients undergoing stand-alone LLIF and found increases of $13.5 \%$ in neuroforamen height, $24.7 \%$ in neuroforamen cross-sectional area, and $33.1 \%$ in central canal diameter. In patients undergoing ALIF, 2 studies have found increases in foramen cross-sectional area at L5-S1 (43.4\% and $67 \%)^{7,28}$ In the setting of spondylolisthesis, additional gains in central canal diameter can be expected as the degree of vertebral body reduction translates into an increase in the canal diameter.

All the patients in our case series showed statistically significant decreases in their VAS and ODI scores on par with those seen after posterior approaches, and no patient required subsequent posterior decompression due to inadequate relief of radiculopathy or neurogenic claudication. Failure of indirect decompression occurs most commonly due to subsidence of the interbody. Strategies to minimize the risk of subsidence include normalizing bone mineral density in osteoporotic patients prior to therapy, avoiding violation of the vertebral endplate during preparation, and using the widest possible graft footprint. ${ }^{17}$

\section{Limitations}

This study has several important limitations. The first is that due to the retrospective nature of this study based on an MIS operative database, generalizability of our treatment strategy is limited since we are unable to provide absolute numbers of how many patients with grade II spondylolisthesis were treated nonoperatively or deferred surgical intervention. Secondly, our strategy is not viable in the setting of osteoporosis, which excluded many patients who underwent preoperative evaluation. Lastly, our overall duration of follow-up is short, with slightly more than half of the patients having undergone clinical and radiographic evaluation at least 1 year after surgery. Clearly, studies with longer-term follow-up are needed to ascertain the efficacy of our treatment strategy outside of the perioperative period.

\section{Conclusions}

MIS ALIF and LLIF are viable strategies for achieving closed reduction of grade II lumbar spondylolisthesis. Our case series shows a high rate of complete reduction, with no occurrence of persistent motor deficits and with low perioperative blood loss and patient length of stay. $\mathrm{Pa}$ tient outcomes showed significant reduction of VAS and ODI scores, and no patient required posterior decompression. Our data provide early clinical evidence that ALIF and LLIF can safely and successfully treat higher-grade spondylolisthesis. Limitations of our study include its retrospective nature and short follow-up. Additional studies are needed to examine the durability of clinical outcomes.

\section{References}

1. Ahmadian A, Deukmedjian AR, Abel N, Dakwar E, Uribe JS: Analysis of lumbar plexopathies and nerve injury after lateral retroperitoneal transpsoas approach: diagnostic standardization. J Neurosurg Spine 18:289-297, 2013

2. Ahmadian A, Verma S, Mundis GM Jr, Oskouian RJ Jr, Smith DA, Uribe JS: Minimally invasive lateral retroperitoneal transpsoas interbody fusion for L4-5 spondylolisthesis: clinical outcomes. J Neurosurg Spine 19:314-320, 2013

3. Benglis DM, Vanni S, Levi AD: An anatomical study of the lumbosacral plexus as related to the minimally invasive transpsoas approach to the lumbar spine. J Neurosurg Spine 10:139-144, 2009

4. Bergey DL, Villavicencio AT, Goldstein T, Regan JJ: Endoscopic lateral transpsoas approach to the lumbar spine. Spine (Phila Pa 1976) 29:1681-1688, 2004

5. Boos N, Marchesi D, Zuber K, Aebi M: Treatment of severe spondylolisthesis by reduction and pedicular fixation. A 4-6year follow-up study. Spine (Phila Pa 1976) 18:1655-1661, 1993

6. Bridwell KH, Lenke LG, McEnery KW, Baldus C, Blanke $\mathrm{K}$ : Anterior fresh frozen structural allografts in the thoracic and lumbar spine. Do they work if combined with posterior fusion and instrumentation in adult patients with kyphosis or anterior column defects? Spine (Phila Pa 1976) 20:14101418,1995

7. Cho W, Sokolowski MJ, Mehbod AA, Denis F, Garvey TA, Perl J, et al: MRI measurement of neuroforaminal dimension at the index and supradjacent levels after anterior lumbar interbody fusion: a prospective study. Clin Orthop Surg 5:49-54, 2013

8. de Kunder SL, van Kuijk SMJ, Rijkers K, Caelers IJMH, van 
Hemert WLW, de Bie RA, et al: Transforaminal lumbar interbody fusion (TLIF) versus posterior lumbar interbody fusion (PLIF) in lumbar spondylolisthesis: a systematic review and meta-analysis. Spine J [epub ahead of print], 2017

9. Epstein NE: More nerve root injuries occur with minimally invasive lumbar surgery: Let's tell someone. Surg Neurol Int 7 (Suppl 3):S96-S101, 2016

10. Goyal N, Wimberley DW, Hyatt A, Zeiller S, Vaccaro AR, Hilibrand AS, et al: Radiographic and clinical outcomes after instrumented reduction and transforaminal lumbar interbody fusion of mid and high-grade isthmic spondylolisthesis. J Spinal Disord Tech 22:321-327, 2009

11. Hu WK, He SS, Zhang SC, Liu YB, Li M, Hou TS, et al: An MRI study of psoas major and abdominal large vessels with respect to the X/DLIF approach. Eur Spine J 20:557-562, 2011

12. Januszewski J, Beckman JM, Bach K, Vivas AC, Uribe JS: Indirect decompression and reduction of lumbar spondylolisthesis does not result in higher rates of immediate and long term complications. J Clin Neurosci 45:218-222, 2017

13. Kasliwal MK, Smith JS, Shaffrey CI, Saulle D, Lenke LG, Polly DW Jr, et al: Short-term complications associated with surgery for high-grade spondylolisthesis in adults and pediatric patients: a report from the Scoliosis Research Society Morbidity and Mortality database. Neurosurgery 71:109116,2012

14. Kwon BK, Hilibrand AS, Malloy K, Savas PE, Silva MT, Albert TJ, et al: A critical analysis of the literature regarding surgical approach and outcome for adult low-grade isthmic spondylolisthesis. J Spinal Disord Tech 18 Suppl:S30-S40, 2005

15. Labelle H, Roussouly P, Chopin D, Berthonnaud E, Hresko T, O'Brien M: Spino-pelvic alignment after surgical correction for developmental spondylolisthesis. Eur Spine J 17:11701176, 2008

16. Lauber S, Schulte TL, Liljenqvist U, Halm H, Hackenberg L: Clinical and radiologic 2-4-year results of transforaminal lumbar interbody fusion in degenerative and isthmic spondylolisthesis grades 1 and 2. Spine (Phila Pa 1976) 31:16931698,2006

17. Le TV, Baaj AA, Dakwar E, Burkett CJ, Murray G, Smith DA, et al: Subsidence of polyetheretherketone intervertebral cages in minimally invasive lateral retroperitoneal transpsoas lumbar interbody fusion. Spine (Phila Pa 1976) 37:12681273,2012

18. Lee SH, Choi WG, Lim SR, Kang HY, Shin SW: Minimally invasive anterior lumbar interbody fusion followed by percutaneous pedicle screw fixation for isthmic spondylolisthesis. Spine J 4:644-649, 2004

19. Lenke LG, Bridwell KH, Bullis D, Betz RR, Baldus C, Schoenecker PL: Results of in situ fusion for isthmic spondylolisthesis. J Spinal Disord 5:433-442, 1992

20. Longo UG, Loppini M, Romeo G, Maffulli N, Denaro V: Evidence-based surgical management of spondylolisthesis: reduction or arthrodesis in situ. J Bone Joint Surg Am 96:53-58, 2014

21. Marchi L, Abdala N, Oliveira L, Amaral R, Coutinho E, Pimenta L: Stand-alone lateral interbody fusion for the treatment of low-grade degenerative spondylolisthesis. Sci World J 2012:456346, 2012

22. Mobbs RJ, Phan K, Malham G, Seex K, Rao PJ: Lumbar interbody fusion: techniques, indications and comparison of interbody fusion options including PLIF, TLIF, MI-TLIF, OLIF/ATP, LLIF and ALIF. J Spine Surg 1:2-18, 2015

23. Mummaneni PV, Bisson EF, Kerezoudis P, Glassman S, Foley $\mathrm{K}$, Slotkin JR, et al: Minimally invasive versus open fusion for Grade I degenerative lumbar spondylolisthesis: analysis of the Quality Outcomes Database. Neurosurg Focus 43(2):E11, 2017

24. Oliveira L, Marchi L, Coutinho E, Pimenta L: A radiographic assessment of the ability of the extreme lateral interbody fusion procedure to indirectly decompress the neural elements. Spine (Phila Pa 1976) 35 (26 Suppl):S331-S337, 2010

25. Park P, Foley KT: Minimally invasive transforaminal lumbar interbody fusion with reduction of spondylolisthesis: technique and outcomes after a minimum of 2 years' follow-up. Neurosurg Focus 25(2):E16, 2008

26. Petraco DM, Spivak JM, Cappadona JG, Kummer FJ, Neuwirth MG: An anatomic evaluation of L5 nerve stretch in spondylolisthesis reduction. Spine (Phila Pa 1976) 21:11331139, 1996

27. Rajakumar DV, Hari A, Krishna M, Sharma A, Reddy M: Complete anatomic reduction and monosegmental fusion for lumbar spondylolisthesis of Grade II and higher: use of the minimally invasive "rocking" technique. Neurosurg Focus 43(2):E12, 2017

28. Rao PJ, Maharaj MM, Phan K, Lakshan Abeygunasekara M, Mobbs RJ: Indirect foraminal decompression after anterior lumbar interbody fusion: a prospective radiographic study using a new pedicle-to-pedicle technique. Spine J 15:817-824, 2015

29. Rodgers WB, Gerber EJ, Patterson J: Intraoperative and early postoperative complications in extreme lateral interbody fusion: an analysis of 600 cases. Spine (Phila Pa 1976) 36:2632,2011

30. Sembrano JN, Tohmeh A, Isaacs R: Two-year comparative outcomes of MIS lateral and MIS transforaminal interbody fusion in the treatment of degenerative spondylolisthesis: part I: clinical findings. Spine (Phila Pa 1976) 41 (Suppl 8):S123-S132, 2016

31. Wang MY, Cummock MD, Yu Y, Trivedi RA: An analysis of the differences in the acute hospitalization charges following minimally invasive versus open posterior lumbar interbody fusion. J Neurosurg Spine 12:694-699, 2010

\section{Disclosures}

Dr. Uribe reports a consultant relationship with and direct stock ownership in NuVasive.

\section{Author Contributions}

Conception and design: Uribe, Xu. Acquisition of data: Bach. Analysis and interpretation of data: Xu. Drafting the article: Xu. Critically revising the article: all authors. Reviewed submitted version of manuscript: Xu. Statistical analysis: Xu. Administrative/technical/material support: Uribe. Study supervision: Uribe.

\section{Correspondence}

Juan S. Uribe: Barrow Neurological Institute, St. Joseph's Hospital and Medical Center, Phoenix, AZ. juansuribe@gmail.com. 